\title{
El plan de apoyo conductual positivo en el primer ciclo de Educación Infantil
}

\author{
Positive behaviour support plan in the first cycle of early childhood \\ education
}

\begin{abstract}
Resumen
Este estudio pretende analizar los efectos que tiene el plan de apoyo conductual positivo sobre las habilidades adaptativas, el comportamiento y el desarrollo socioemocional del alumnado del primer ciclo de Educación Infantil. Se evaluó la primera variable utilizando el Sistema de Evaluación de la Conducta Adaptativa (ABAS-II) y las dos últimas, el Infant Toddler Social Emotional Assessment (ITSEA). Los resultados mostraron una disminución estadísticamente significativa del comportamiento externalizante $(Z=-2,418, p=, 016)$, la falta de regulación $(Z=-2,767, p=, 006)$ y otras conductas poco adaptativas $(Z=-2,357, p=, 018)$, así como un incremento estadísticamente significativo de la competencia $(Z=-2,959, p=, 003)$, la comunicación $(Z=-$ $3,576, p=, 000)$, el desarrollo social $(Z=-3,622, p=, 000)$, el ocio $(Z=-3,542, p=, 000)$, el autocuidado $(Z=-3,361, p=, 000)$, la autodirección $(Z=-3,575, p=, 000)$ y la adaptación a la escuela $(Z=-3,662, p=, 000)$. Son resultados que muestran la importancia que tiene crear un entorno escolar accesible y la necesidad de apoyar intencionadamente el desarrollo de las habilidades adaptativas en esta etapa educativa.
\end{abstract}

\section{Palabras clave}

Plan de apoyo positivo a la conducta, conducta desafiante, Educación Infantil, educación inclusiva, intervención preventiva y proactiva, desarrollo socioemocional, habilidades adaptativas.

\begin{abstract}
This study aims to analyse the effects of the positive behavioral support plan on the adaptive skills, behavior and social-emotional development of students in the first cycle of early childhood education. The first variable was evaluated using the Adaptive Behavior Assessment System (ABAS-II) and the last two, the Infant Toddler Social Emotional Assessment (ITSEA). The results showed a statistically significant decrease in externalizing behavior $(Z=-2,418, p=, 016)$, lack of regulation $(Z=-2,767, p=, 006)$ and other poorly adaptive behaviors $(Z=-2,357, p=, 018)$, as well as a statistically significant increase in competence $(Z=-2,959, p=, 003)$, communication $(Z=-3,576, p=, 000)$, social development $(Z=-3,622, p=, 000)$, leisure $(Z=-3,542, p=, 000)$, self-care $(Z=-$ $3,361, p=, 000)$, self-direction $(Z=-3,575, p=, 000)$ and adaptation to school $(Z=-3,662$, $p=, 000)$. These results show the importance of creating an accessible school environment and the need to intentionally support the development of adaptive skills at this educational stage.
\end{abstract}

\section{Keywords}

Positive support plan for behavior, challenging behavior, early childhood education, inclusive education, preventive and proactive intervention, social-emotional development, adaptive skills.

\section{Asunción González del Yerro Valdés}

<asuncion.gonzalezdelyerro@ uam.es>

Universidad Autónoma de Madrid. España

Laura Escribano Burgos

<lescribanoalanda@yahoo.es>

Asociación Alanda. España

\section{María Luisa de Antonio \\ Pérez \\ <escuelamagos@gmail.com> \\ Escuela Infantil Magos. España}

\section{Ana Sánchez Rodríguez} <aniitasan7@gmail.com>

Escuela Infantil Magos. España

Patricia Ruiz Leal

<ruiz.leal.patri@gmail.com>

Escuela Infantil Magos. España

Marta Arellano Lozano <marty_arelo@hotmail.com>

Escuela Infantil Magos. España

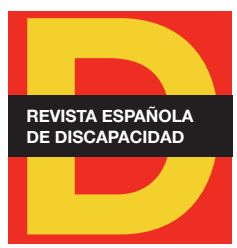

Para citar:

González del Yerro, A. et al. (2019): "El plan de apoyo conductual positivo en el primer ciclo de Educación Infantil". Revista Española de Discapacidad, 7 (I): 67-86.

Doi: <https://doi.org/10.5569/23405104.07.01.04>

Fecha de recepción: 05-02-2018 Fecha de aceptación: 03-04-2019

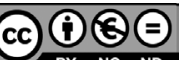




\section{Introducción}

La Educación Infantil es una de las etapas educativas en las que las conductas desafiantes (tales como mordiscos, patadas, empujones, rabietas, lanzar objetos, etc.) son más frecuentes. La prevalencia de desórdenes conductuales diagnosticados en la población de entre dos y cinco años oscila entre un $10 \%$ y un $21 \%$, proporciones que las circunstancias socioeconómicas adversas elevan hasta el $23 \%$ y el $33 \%$, y las condiciones personales asociadas a discapacidad, hasta el $33 \%$ y el $50 \%$ (según la revisión de Fox y Hemmeter, 2009).

En la actualidad, se sabe que este tipo de comportamientos disruptivos afecta negativamente al aprendizaje, al desarrollo socioemocional y al ajuste escolar (Powell et al., 2006). Sin embargo, la consideración de que estas conductas son comportamientos propios de la infancia y la suposición de que terminan superándose en años posteriores retrasó durante un tiempo la aplicación de programas conductuales preventivos en esta primera etapa de la vida (Campbell et al., 2000). No obstante, esta suposición no resulta siempre cierta. La evidencia empírica que revisan Campbell et al. (2000) muestra que del $50 \%$ al $60 \%$ de los niños que tienen conductas externalizantes a los tres años continúa manteniéndolas en el futuro (Richman et al., 1982), que difícilmente remiten cuando son finalmente diagnosticadas en la edad escolar y que, con frecuencia, los comportamientos problemáticos iniciales constituyen precursores de las conductas antisociales más serias que aparecen posteriormente en la adolescencia y en la juventud (Bennett et al., 1998). Al parecer, cuanto más precoces y persistentes son estas conductas, más probable es que se mantengan en la edad escolar (Moffitt, 1993) (a quien citan Shaw y Taraban, 2017).

Las razones por las que solo algunos niños consiguen superar estos comportamientos problemáticos parecen asociarse tanto a factores personales, como a variables situadas en los distintos niveles del entorno (Campbell et al., 2000; Justicia et al., 2006; Marchand et al., 2002; Tremblay et al., 2005). Entre los primeros, suelen destacarse niveles elevados de enfado y afecto negativo, impulsividad, hiperactividad, problemas de atención y falta de empatía. Y, entre los segundos, con relación al contexto familiar, estrategias ineficaces de disciplina, falta de afecto e implicación, establecimiento de vínculos de apego inseguro o desorganizado, pautas conflictivas de interacción, ausencia de modelos adecuados, altos niveles de estrés, problemas de salud mental, ejemplos de comportamiento antisocial en la historia previa de la madre, conflictos familiares serios y la maternidad precoz; factores a los que se suman igualmente los situados en el macrosistema, por ejemplo, la exposición a las situaciones violentas que presentan los medios de comunicación y la propia realidad social, las políticas educativas que basan los programas de disciplina en la imposición de sanciones, y la carencia de una educación personalizada que responda a las necesidades del alumnado.

Con relación al contexto escolar, las investigaciones desarrolladas en el siglo pasado causaron cierta alarma pues asociaban las conductas disruptivas al tiempo de permanencia en los centros. Algunos autores observaron que los bebés que, durante el primer año de vida, pasaban más de veinte horas semanales en las guarderías creaban un vínculo de apego más inseguro con sus padres y, en años posteriores, cooperaban menos con el adulto, eran más activos motrizmente y presentaban frecuencias de conductas agresivas mayores que sus compañeros (Belsky, 1988). Estudios posteriores matizaron este dato al mostrar que esta relación solo aparecía en los centros que impartían una educación de baja calidad, pues el alumnado que asistía a escuelas de alta calidad participaba más, mostraba un interés mayor y menos problemas de conducta 
(Hausfather et al., 1997). Entre las variables que reducían la aparición de comportamientos problemáticos se encontraban la cantidad de interacción verbal entre profesores y niños (de tres años) (Phillips et al., 1987) y la calidad de la relación que establecían entre ellos (a los cuatro años) (Peisner-Feinberg et al., 2001).

El efecto que tienen los factores ambientales y la creencia de que las conductas son tanto más maleables cuanto antes se inicie la intervención condujeron, finalmente, a aplicar programas preventivos desde los primeros años de vida (Campbell et al., 2000; Shaw y Taraban, 2017). La mayoría de estos programas se han desarrollado en el contexto familiar y han mostrado cómo mejorar significativamente la conducta en los primeros años de vida, mediante intervenciones dirigidas a mejorar la calidad de vida familiar (Shaw et al., 2009) y las habilidades parentales, especialmente, la habilidad para interactuar con sus hijos y para afrontar las conductas desafiantes (Sanders, 2008; Schuhmann et al.,1998; Webster-Stratton, 1982).

En las últimas décadas, se ha empezado a aplicar con éxito el plan de apoyo conductual positivo (PACP) en la escuela infantil. Se trata de una intervención de carácter proactivo que pretende prevenir la aparición de las conductas desafiantes mediante la enseñanza de habilidades y la creación de contextos que respondan a las necesidades del alumnado. El PACP suele incluir tres niveles de práctica: a) un nivel universal que se dirige a todo el alumnado, b) un segundo nivel centrado en los estudiantes que tienen riesgo de desarrollar conductas desafiantes, y c) un tercer nivel diseñado para quienes ya manifiestan comportamientos problemáticos. La "Pirámide de la enseñanza" (Fox et al., 2003) constituye un ejemplo de este tipo de programas caracterizado por incluir en el nivel preventivo universal dos tipos de prácticas educativas (Powell et al., 2006). El objetivo de la primera es asegurar la formación de relaciones positivas entre todos los miembros de la comunidad educativa, y el de la segunda, la creación de entornos que generen bienestar y favorezcan el aprendizaje y el desarrollo.

El tercer nivel de la pirámide se dirige al alumnado que tiene más riesgo de presentar conductas desafiantes y consiste en la enseñanza explícita de habilidades sociales. En la actualidad, tiende a afirmarse la necesidad de extender al nivel universal la enseñanza explícita de estas habilidades en la Educación Infantil (Fox y Hemmeter, 2009). España (entre otros países) ya se había enfrentado al reto de apoyar el desarrollo de la competencia social en esta etapa educativa. Monjas y González (1998) desarrollaron el "Programa de Enseñanza de Habilidades de Interacción Social" en un colegio de Cogeces del Monte (Valladolid) con el fin de integrar la enseñanza de habilidades sociales en el currículo y, más recientemente, Justicia-Arráez et al. (2015) aplicaron el programa "Aprender a convivir" que se desarrolla en 24 sesiones de 45 minutos y consta de cuatro bloques: 1) las normas y su cumplimiento, 2) sentimientos y emociones, 3) habilidades de comunicación y 4) ayuda y cooperación, con los que consiguieron mejorar significativamente la cooperación, interacción e independencia social del alumnado y reducir de forma igualmente significativa las conductas problemáticas (Pichardo et al., 2016).

El cuarto nivel de la pirámide se centra en el alumnado que ya ha comenzado a presentar conductas desafiantes; se trata de una actuación que, siguiendo los principios del plan centrado en la persona, se dirige a mejorar su experiencia vital y su calidad de vida, a apoyar el desarrollo de sus habilidades comunicativas y sociales, su integración en el medio y su adaptación al entorno; para ello, junto a la familia y a otras personas relevantes en la vida del individuo se planifica y desarrolla un plan de intervención basado en un análisis funcional encaminado a identificar la función de las conductas desafiantes, y los factores personales y ambientales que influyen en su aparición (Fox et al., 2003). 
La aplicación de este modelo basado en la Pirámide de la enseñanza se ha mostrado eficaz para prevenir la aparición de conductas desafiantes y para mejorar el desarrollo socioemocional del alumnado de Educación Primaria (Escribano et al., 2014) y del segundo ciclo de Educación Infantil (Benedict et al., 2007; Conroy et al., 2005; Fox et al., 2002; Fox y Hemmeter, 2009; González del Yerro et al., 2010; Powell y Dunlap, 2009). Sin embargo, su aplicación en el primer ciclo es todavía muy escasa (Dunlap y Fox, 2009) o no se acompaña siempre de un análisis de los efectos que tiene esa práctica educativa en el alumnado (Fox et al., 2001) o, en todo caso, requiere un análisis mayor dada la gran heterogeneidad de formas que adopta la educación que en este primer ciclo se imparte (Dunlap et al., 2006; Fox et al., 2002; Hemmeter et al., 2007).

Con el fin de responder a las necesidades del alumnado de una escuela infantil inclusiva, las autoras Laura Escribano y María Luisa de Antonio han desarrollado, tras más de veinte años de colaboración, una metodología que comparte con la pirámide de la enseñanza un gran número de prácticas educativas (cuya publicación se encuentra en fase de preparación) que vamos a resumir siguiendo los niveles que diferencia la Pirámide de la Enseñanza (ilustrándolo con ejemplos para facilitar su comprensión):

\section{Establecimiento de relaciones sociales positivas}

La escuela entiende la educación como una práctica compartida y colabora con las familias con el fin de asegurar el bienestar físico y emocional del alumnado. Para ello, intentan establecer acuerdos sobre criterios y pautas de actuación, se intercambian con frecuencia información sobre temas de interés (el proyecto educativo, las necesidades del día a día, el alumnado, recursos del entorno, pautas de crianza, etc.) utilizando diferentes canales de comunicación (reuniones, mails, página web, teléfono, intercambios comunicativos informales, etc.) e invitando a las familias a participar en las actividades de la escuela (donde les enseñan canciones, aparatos curiosos (telescopios, cámaras de fotos, etc.), les acompañan en las salidas al entorno, actúan como cómplices en los proyectos, colaboran con otros agentes del entorno en actuaciones de distinta índole, etc.).

Igualmente, el equipo educativo orienta a las familias sobre cómo apoyar el aprendizaje y el desarrollo y facilita la comunicación entre adultos y menores utilizando distintos procedimientos como, por ejemplo, el Cuaderno Viajero, la introducción de apoyos visuales en la información que se envía a las familias (horarios, cartas, anuncios, informes de evaluación, etc.), el amigo Mariete (un muñeco al que invitan a las casas a convivir y compartir experiencias que posteriormente relatan en la clase con la ayuda de pictogramas o fotos), entre otros.

Las profesoras establecen con cada alumno o alumna una relación cercana y respetuosa, basada en la concepción del niño como sujeto, lo que les conduce a actuar para que se perciban a sí mismos y a los demás como tales: a crear un contexto accesible que todos puedan comprender, a dar oportunidades para elegir y a dejar que sus decisiones configuren una parte del día a día, a reservar a cada uno ciertos lugares en la escuela para que los sientan como propios y a enseñarles a respetar y a que se respete ese espacio (por ejemplo, eligen un dibujo para señalar su sitio en la asamblea y así se evita, además, que cuando lleguen a ella se vaya estrechando tanto el círculo que terminen sentándose unos sobre otros o en la fila de atrás). Todas las prácticas de la escuela que se expondrán a continuación se basan en esta idea.

\section{Creación de un entorno que apoya el aprendizaje y el desarrollo}

La escuela crea un entorno que ofrece seguridad; para ello, desarrollan prácticas propias del Programa de Estructuración Ambiental para Niños con Autismo (PEANA) propuesto por Tamarit et al. (1990), caracterizado 
por utilizar apoyos visuales para delimitar y marcar lugares relevantes de la escuela (como, por ejemplo, las aulas, el contenido de los armarios, la percha en la que colgar el abrigo, etc.), por establecer rutinas estables y horarios visuales, que utilizan para informarles sobre las rutinas y las actividades que se van a desarrollar en el día, para anticipar su inicio y su fin, y los posibles cambios que pudieran ocurrir e, igualmente, por darles esa misma información sobre las actividades que se desarrollarán en los proyectos.

El equipo educativo procura que el alumnado perciba la escuela como una extensión del contexto familiar. Para ello, exponen fotos de sus familias en la pared del aula, las invitan, como hemos visto, a participar en diferentes actividades y unos dibujos grandes de "papás" y "mamás" (cuyo número se ajusta a las estructuras familiares presentes en la clase) dan la bienvenida en la pared de las aulas.

Su programación se estructura en proyectos seleccionados en función de los intereses del grupo; en ellos, se utiliza el juego como actividad natural de aprendizaje y se desarrollan actividades atractivas (canciones, guiñoles, cuentos, salidas al entorno, elaboración de materiales, etc.) que adquieren sentido por estar enmarcadas en proyectos sobre el mundo de la fantasía, el arte o el universo y que, consecuentemente, invitan a elaborar barcos de piratas, garfios, pañuelos, trajes espaciales, escafandras, cohetes, salidas al entorno, etc., ajustando siempre los parámetros de la actividad (ej., liderazgo, agrupamientos y, especialmente, la duración) a las necesidades del alumnado.

La escuela aplica técnicas de apoyo positivo a la conducta: 1 ) uso frecuente del refuerzo positivo (aunque se utiliza de una forma especial pues se promueve que sean ellos mismos los que valoren su propia actuación sustituyendo, por ejemplo, el habitual "iQué contenta estoy porque has comido!" (que genera una dependencia del adulto) por un "¡Qué contento estás porque has comido!", que les reconoce como sujetos responsables de la valoración de su propia conducta; 2) explicación detallada y en positivo de las conductas esperadas (por ejemplo, en el teatro, "se habla bajito") que, cuando es necesario se practican como veremos posteriormente; 3 ) establecimiento de normas (una cada vez) explicadas con claridad con la ayuda de imágenes y recordadas con frecuencia tras dar retroalimentación (así, por ejemplo, en la hora de la comida, la profesora felicita al grupo por lo bien que están sentados esperando la comida y les pregunta cómo quieren la estrella que dibujará (ante todos en un gran papel próximo al dibujo que representa la norma) "grande como una casa o pequeña como una hormiga"; 4) se redirige la conducta inadecuada invitando a realizar una conducta alternativa (ej., "mejor jugamos con la pelota") en lugar de emplear el "no" que se considera que "imanta", es decir, que genera una tendencia a repetir lo prohibido.

\section{Apoyo al desarrollo de habilidades}

El desarrollo comunicativo y lingüístico es la prioridad máxima de la escuela (en sintonía con la psicología soviética); junto a este objetivo, la escuela apoya, fundamentalmente, el desarrollo emocional, social y el de la autodeterminación. Lo hace (entre otros) centrando la intervención en las actividades y rutinas que se desarrollan a lo largo del día. En ellas se brindan numerosas oportunidades para elegir, relacionarse, expresarse y escuchar. Las profesoras animan la expresión verbal, gastan bromas, les piden sugerir ideas, llamar a algún personaje, ayudarles a encontrar algún material que simulan haber olvidado, a anticipar detalles de los cuentos, etc., y les ofrecen numerosas oportunidades para elegir y expresar su opinión utilizando en ocasiones procedimientos formales de votación; les permiten que su voz -(que quienes lo precisan expresan con diferentes sistemas de comunicación, como signos o pictogramas)- determine lo que se va a hacer en el aula y les enseñan a aceptar las preferencias de los demás cuando la propia no coincide con las de la mayoría. 
Las profesoras son conscientes de la importancia que tiene su propia forma de hablar en el desarrollo lingüístico y se expresan con claridad, de forma pausada, marcada entonación (cuando la ocasión lo requiere) y utilizando oraciones completas que van enriqueciendo con un vocabulario diverso procedente de cuentos, juegos, canciones, proyectos y de la propia realidad que invitan a conocer. Con el fin de eliminar las barreras que dificultan el uso del lenguaje, utilizan el Programa de Comunicación Total de Benson Schaeffer (Schaeffer et al., 2005), y otros apoyos visuales tales como pictogramas, fotos y dibujos.

Para apoyar el desarrollo emocional, las profesoras verbalizan las distintas fases implicadas en la autorregulación emocional: identifican las emociones, reconocen sus causas, la acción que podrían realizar para recobrar un estado emocional positivo (cuando la ocasión lo requiere) y el modo de expresión, por ejemplo, "lloras porque estás cansado y tienes sueño, claro, mejor... "quiero dormir"', aunque no siempre la solución puede ser tan inmediata y se verbaliza entonces lo que se hará hasta poder alcanzarla (ej., "lloras porque estás cansado y tienes sueño, claro..... ahora vamos a comer y luego a dormir"). De esta manera, los menores se sienten escuchados y comprendidos y ello les ayuda a gestionar sus propias emociones. Las profesoras emplean, del mismo modo, apoyos visuales tales como "la mamá de bolsillo", un recortable dibujado que pueden llevar en el bolsillo de su babi para apoyar la autorregulación emocional.

Con el fin de facilitar la adquisición de las habilidades sociales (entre las que destaca el uso de la palabra para resolver conflictos) se utiliza el mismo tipo de andamiaje (Wood et al., 1976) descrito previamente. Las profesoras verbalizan todo aquello que intuyen que los menores querrían expresar si pudieran empleando un lenguaje ajustado al nivel de cada uno ("dame el coche"), así como la instrucción explícita, la mediación y el modelado de adultos e iguales, tanto en situaciones naturales, como en el "juego simbólico", en el que un pequeño grupo liderado por la maestra representa una situación que incluye una habilidad social (como, por ejemplo, compartir).

Del mismo modo, junto a tiempos dedicados al juego libre, se dedican tiempos a enseñar otros como, por ejemplo, tula (en una versión simplificada), el tren, a la zapatilla por detrás, "que viene la bruja o el elefante, el cocodrilo o cualquier otro personaje"; con este último toman conciencia de sus propios miedos -requisito necesario para superarlos, pues solo cuando el individuo toma conciencia de sus procesos psicológicos elementales, los domina, consigue someter al control voluntario, lo que antes se encontraba dominado por los estímulos del entorno (Vygotski, 1993). Son juegos que aunque tienen reglas muy primitivas (pues a veces no se diferencian apenas de la propia acción del juego) conservan el papel configurador (o de cohesión) de pequeñas sociedades infantiles (Linaza y Maldonado, 1987), a los que se aprende a jugar jugando, participando en la actividad conjunta, en la que en primer lugar se imitan las conductas de los otros y poco a poco se va alcanzando una comprensión mayor de la dinámica del juego, como si fueran los juegos de reglas que Piaget (1984) describe en edades posteriores.

En la escuela, los menores aprenden asimismo los guiones propios de los contextos sociales en los que van a participar (junto a las normas, solo una), por ejemplo, se enseña el guión propio de los museos y su norma ("los cuadros se ven con los ojos"), creando entre todos un museo en el propio aula y todos los materiales que habitualmente se encuentran en él (incluidas las entradas) y, tras informarles por adelantado, se simula visitar el museo antes de acudir al museo de la ciudad.

Se ofrecen igualmente oportunidades para practicar el autocontrol; se incluyen, por ejemplo, tiempos de espera breves (ej., les explican algo antes de coger los juguetes) para que aprendan a frenar su impulsividad, 
se desarrollan actividades que requieren prestar atención (como, por ejemplo, los cuentos, las canciones que enseñan las familias, el procedimiento formal de las votaciones), actividades de relajación que tienen sentido en el proyecto tales como "andar como en la luna" en el proyecto del espacio, a aceptar las preferencias de los demás cuando no coinciden con las propias, a utilizar palabras o signos para expresar lo que quieren y tienen los demás, etc.

Este estudio tiene dos objetivos: 1) analizar la mejoría que experimenta el alumnado de dos a tres años en la conducta, el desarrollo socioemocional y, de forma general, en las habilidades adaptativas, tras recibir durante un curso escolar una práctica educativa basada en el PACP y 2) examinar la relación existente entre la conducta y los ámbitos del desarrollo que apoya la escuela de forma prioritaria: la comunicación y el lenguaje, la autodeterminación, y el desarrollo socioemocional.

Se plantean las siguientes hipótesis de partida:

1. Al final del curso escolar, se observará una mejoría significativa en el desarrollo socioemocional, la conducta y, en general, las habilidades adaptativas de los niños y las niñas del aula dos-tres.

2. Existe una relación significativa entre las conductas problemáticas y los ámbitos del desarrollo que la escuela apoya de modo prioritario (comunicación y lenguaje, desarrollo social y autodeterminación).

\section{Método}

\section{Participantes}

La investigación se desarrolló en el aula de dos a tres años de una escuela inclusiva seleccionada mediante un muestreo intencional. En él, participaron las diez niñas y los ocho niños que conformaban la clase. Dos de ellos recibían, además, el apoyo de una asociación especializada en dificultades en el desarrollo comunicativo y de la conducta que aplica, entre otros, el programa Hanen y el Programa GOMA (Escribano, 2014) que es un programa dirigido a las familias que está basado en el PACP. El aula está siempre atendida por una tutora y una profesora de apoyo. Todas las familias presentaban un nivel socioeconómico medio; tres de ellas ofrecían a sus hijos una educación bilingüe, aunque una tuvo que interrumpirla a mitad de curso por tener que regresar a su país de origen. Los padres o las madres de las diez niñas y de cuatro de los niños tenían una formación universitaria.

\section{Diseño de la investigación}

Se trata de un estudio cuasi-experimental $A B$, en el que la variable independiente es la práctica educativa que se desarrolla en la escuela infantil previamente descrita y las variables dependientes, el desarrollo socioemocional, las conductas problemáticas, y las habilidades adaptativas: comunicación y lenguaje, desarrollo social, autocuidado, autodirección, ocio y adaptación a la escuela.

\section{Instrumentos}

Para evaluar el desarrollo socioemocional y las conductas problemáticas se utilizaron 69 ítems de los 166 que componen la escala Infant Toddler Social Emotional Assessment (ITSEA) (Carter et al., 2003). Esta escala 
evalúa la competencia socioemocional y los comportamientos problemáticos que experimentan los niños de 12 a 36 meses de edad. La escala se encuentra dividida en cinco dominios que, a su vez, se dividen en las dimensiones que exponemos en la Tabla 1, junto al coeficiente de Cronbach (p) que cada una alcanzó, el número de ítems utilizados en cada dominio y un ejemplo de los ítems que constituyen cada una de las dimensiones.

Tabla 1. Dominios y número de ítems utilizados en cada dimensión del ITSEA

\begin{tabular}{|c|c|c|c|}
\hline Dominio & Dimensión & Ejemplo & $\mathbf{n}$ \\
\hline \multirow{3}{*}{$\begin{array}{l}\text { Comportamiento } \\
\text { externalizante } \\
(p=.966, n=20)\end{array}$} & Impulsividad & Es incansable. No puede permanecer quieto & 4 \\
\hline & Agresión /desafío & Rompe o estropea cosas a propósito & 11 \\
\hline & Agresión a niños & Pega, empuja o muerde a otros niños & 5 \\
\hline \multirow{4}{*}{$\begin{array}{l}\text { Comportamiento } \\
\text { internalizante } \\
(p=.916, n=16)\end{array}$} & Ansiedad-temor & Le dan miedo algunas cosas & 4 \\
\hline & Depresión & Parece triste sin razón & 6 \\
\hline & Resistencia a la novedad & Se muestra tímido ante desconocidos & 2 \\
\hline & Malestar ante la separación & Llora al separarse de sus padres & 4 \\
\hline \multirow{4}{*}{$\begin{array}{l}\text { Falta de regulación } \\
(p=.947, n=16)\end{array}$} & Comida & Escupe lo que come & 4 \\
\hline & Sueño & Le cuesta conciliar el sueño & 1 \\
\hline & Estímulos sensoriales* & $\begin{array}{l}\text { Le molesta el tacto, el olor, algunos sonidos, luces, } \\
\text { texturas... }\end{array}$ & 1 \\
\hline & Afecto negativo & Se queja aunque no esté cansado & 10 \\
\hline \multirow{3}{*}{$\begin{array}{l}\text { Otras conductas } \\
(p=.927, n=17)\end{array}$} & Conductas atípicas & Repite un determinado movimiento una y otra vez & 4 \\
\hline & Conductas poco adaptativas & Come o bebe lo que no debe (ej., pintura, papel, etc.) & 8 \\
\hline & Conductas con significado clínico & Se hace daño a propósito & 5 \\
\hline La competencia & Atención & Juega con los juguetes durante cinco minutos o más & 5 \\
\hline \multirow[t]{5}{*}{$(p=.973, n=37)$} & Cumplimiento & Intenta hacer lo que le pides & 8 \\
\hline & Empatía & Se entristece cuando alguien se hiere & 7 \\
\hline & Imitación y juego & Hace que alimenta a muñecas o a animales & 6 \\
\hline & Motivación & Se alegra cuando le sale algo bien & 6 \\
\hline & Relaciones entre iguales & Juega bien con otros niños & 5 \\
\hline
\end{tabular}

p: Coeficiente de Cronbach

* El ítem de esta dimensión integra varios de la escala original.

Fuente: elaboración propia.

Este instrumento requiere indicar la frecuencia con la que un alumno presenta la conducta que describe cada ítem utilizando una escala Likert (1: muy infrecuente, 5: muy frecuente).

Igualmente, se pidió al equipo docente describir las conductas que le preocupaban de cada menor, y la frecuencia con la que esas conductas se presentaban en una escala Likert (1: muy infrecuente, 5: muy frecuente).

Para evaluar las habilidades adaptativas se utilizó el Sistema de Evaluación de la Conducta Adaptativa (ABAS-II) (Montero y Fernández-Pinto, 2013) (en su versión para profesores); el instrumento evalúa las habilidades que mostramos en la Tabla 2, junto al Coeficiente Cronbach (que calculamos para comprobar su fiabilidad pues se obtuvieron únicamente las puntuaciones brutas), y un ejemplo de los ítems que incluye. Se consideró que la autodirección evaluaba la autodeterminación. 


Tabla 2. Habilidades adaptativas evaluadas con el ABAS-II
\begin{tabular}{|l|l|}
\hline Habilidad & Ejemplo \\
\hline $\begin{array}{l}\text { Comunicación } \\
(p=.971, n=23)\end{array}$ & Señala los objetos de una habitación cuando se le pide (por ejemplo, "señala la mesa"). \\
\hline $\begin{array}{l}\text { Social } \\
(p=.920, n=24)\end{array}$ & Corre a saludar a familiares y a amigos. \\
\hline $\begin{array}{l}\text { Ocio } \\
(p=.922, n=22)\end{array}$ & Juega con el mismo juguete o juego durante al menos un minuto. \\
\hline $\begin{array}{l}\text { Autocuidado } \\
(p=.778, n=20)\end{array}$ & Sostiene y bebe de un tazón. \\
\hline $\begin{array}{l}\text { Autodirección } \\
(p=.938, n=24)\end{array}$ & Escoge la comida o merienda que quiere cuando se le da a elegir. \\
\hline $\begin{array}{l}\text { Vida en la escuela } \\
(p=.856, n=23)\end{array}$ & Señala el lugar en el que se encuentran guardadas sus cosas en la clase o en la escuela infantil. \\
\hline
\end{tabular}

Fuente: elaboración propia.

Cada uno de estos ítems se responde con una escala Likert (1: nunca cuando es necesario, 3: siempre cuando es necesario) que ofrece, asimismo, la posibilidad de responder "no es capaz" y de indicar que la respuesta dada es una suposición.

\section{Procedimiento}

La investigadora principal se puso en contacto con la escuela debido al prestigio que ya tiene su práctica educativa y pidió a las profesoras que atienden el aula que evaluaran a los menores utilizando los procedimientos de evaluación previamente señalados un mes después de iniciar el "curso escolar" y quince días antes de que finalizara.

Se debe señalar que este estudio se enmarca en un proyecto más amplio de carácter científico y académico que tiene como fin diseñar un programa de formación de carácter audio-visual centrado en el proyecto educativo de la escuela, y analizar cualitativa y cuantitativamente los efectos que tiene su práctica educativa sobre el desarrollo infantil y los programas de intervención en contextos naturales que en ella se desarrollan. Por este motivo, la dirección de la escuela, la representante de la asociación que en ella trabaja y la investigadora principal firmaron un acuerdo al iniciar el curso en el que se especificaban los objetivos del proyecto, se dejaba constancia de la autorización de las familias, del compromiso de confidencialidad de las investigadoras y de las normas que debían regir el uso de los materiales que se elaboraran.

El proyecto requería que una persona externa fuera periódicamente a la escuela a observar, realizar grabaciones y entrevistas. Por todo ello, las maestras no conocían con precisión para qué se iban a utilizar las escalas que para este estudio debían completar.

\section{Análisis de resultados}

El análisis estadístico de los datos se realizó con el programa Statistical Package for the Social Sciences (SPSS) (versión 24). Se utilizó la prueba no paramétrica de Wilcoxon para determinar si había diferencias estadísticamente significativas entre las puntuaciones obtenidas al inicio y al final de curso en las habilidades adaptativas y en el desarrollo socioemocional (evaluadas con el ITSEA y el ABAS-II, respectivamente), así como en la frecuencia de conductas observadas por las maestras, que se analizaron igualmente cualitativamente. 
Del mismo modo, se utilizó el coeficiente de Spearman $\rho$ (rho) para calcular las correlaciones existentes entre las puntuaciones medias de las puntuaciones obtenidas al inicio y al final del curso en los dominios (correspondientes a las conductas problemáticas) y el desarrollo de las habilidades adaptativas: comunicación y lenguaje, desarrollo social y autodirección.

\section{Resultados}

Al inicio de curso, el equipo docente de la escuela indicó que el 58,8\% de los menores mostraban con frecuencia conductas problemáticas tales como pegar, arrebatar juguetes, meterse las manos en la boca, intolerancia a la frustración, rabietas, obsesiones, fijaciones y falta de regulación. Al final de curso, el porcentaje del alumnado que presentaba estas conductas se redujo al 35,3\%, y la frecuencia de estos comportamientos disminuyó de forma estadísticamente significativa $(Z=-2.825, p=.005)$. Los instrumentos formales de evaluación utilizados corroboraron estos datos. Como puede observarse en la Tabla 3, la Prueba de Wilcoxon mostró un decremento estadísticamente significativo en el comportamiento externalizante $(Z=-2.418, p=$ .016), la falta de regulación $(Z=-2.767, p=.006)$ y otras conductas poco adaptativas $(Z=-2.357, p=.018)$.

Tabla 3. Frecuencia de conductas y puntuaciones obtenidas en los dominios del ITSEA al inicio y al final de curso
\begin{tabular}{|l|c|c|c|c|c|c|}
\hline \multirow{2}{*}{ Variables } & Puntuaciones & Inicio & Final & \multicolumn{2}{c|}{ Wilcoxon } \\
\cline { 2 - 8 } & Mín. & Máx. & M (DT) & M (DT) & Z & Sig. \\
\hline Frecuencia conductas & 1 & 5 & $2.71(2.44)$ & $1.18(1.74)$ & -2.825 & .005 \\
\hline C. Externalizante & 1 & 5 & $2.00(.91)$ & $1.63(.74)$ & -2.418 & .016 \\
\hline C. Internalizante & 1 & 5 & $2.23(.78)$ & $1.90(.44)$ & -1.529 & .126 \\
\hline Falta de regulación & 1 & 5 & $2.56(1.04)$ & $1.72(.60)$ & -2.767 & .006 \\
\hline Otras conductas & 1 & 5 & $1.68(.76)$ & $1.29(.36)$ & -2.357 & .018 \\
\hline
\end{tabular}

Fuente: elaboración propia.

Sin embargo, la disminución de la puntuación que experimentó el comportamiento internalizante al final de curso no fue estadísticamente significativa $(Z=-1.529, p=.126)$.

La Tabla 4 muestra los cambios que sufrieron las puntuaciones obtenidas en las dimensiones que componen los dominios referidos a las conductas. Con respecto al comportamiento externalizante, las puntuaciones obtenidas en impulsividad $(Z=-3.314, p=.001)$ y en agresión a niños $(Z=-2.178, p=.029)$ al final de curso fueron significativamente inferiores a las alcanzadas al inicio; el decremento que experimentó la agresión ( $Z$ $=-1.250, p=.211$ ) no fue, sin embargo, estadísticamente significativo.

Con relación al comportamiento internalizante, la puntuación obtenida en la dimensión ansiedad-temor al final de curso fue significativamente inferior a la obtenida al inicio $(Z=-2.357, p=.018)$, sin embargo, la disminución que experimentó la depresión-resistencia a la novedad al final de curso no alcanzó significación estadística. 
Por su parte, en las dimensiones que componen la falta de regulación, se observó que las diferencias entre las puntuaciones obtenidas al inicio y al final de curso fueron estadísticamente significativas en tres dimensiones: la comida $(Z=-2.603, p=, 009)$, el afecto negativo $(Z=-2.564, p=.010)$ y la sensibilidad sensorial ( $Z=-2.401, p=.016)$; en la cuarta (referida a las dificultades para conciliar el sueño) no se observó un decremento estadísticamente significativo $(Z=-1.869, p=.062)$.

Tabla 4. Puntuaciones alcanzadas en el ITSEA al inicio y al final del curso
\begin{tabular}{|l|l|c|c|c|c|c|c|}
\hline \multirow{2}{*}{ Dominios } & \multirow{2}{*}{ Dimensiones } & Puntuación & Inicio & Fin & \multicolumn{2}{c|}{ Wilcoxon } \\
\cline { 2 - 9 } & & Mín. & Máx. & M (DT) & M (DT) & Z & p \\
\hline \multirow{3}{*}{ C.Externalizante } & Impulsividad & 1 & 5 & $2.2(1.1)$ & $1.6(1.0)$ & -3.314 & .001 \\
\cline { 2 - 9 } & Agresión & 1 & 5 & $1.9(.8)$ & $1.7(.7)$ & -1.250 & .211 \\
\cline { 2 - 9 } & Agresión a niños & 1 & 5 & $2.1(1.2)$ & $1.7(.8)$ & -2.029 & .042 \\
\hline \multirow{3}{*}{ C.Internalizante } & Ansiedad-Temor & 1 & 5 & $2.4(.8)$ & $1.9(.6)$ & -2.357 & .018 \\
\cline { 2 - 9 } & Depresión-R.Novedad & 1 & 5 & $2.1(.9)$ & $1.9(.5)$ & -.832 & .406 \\
\hline \multirow{5}{*}{ Falta de regulación } & Comida & 1 & 5 & $2.0(.9)$ & $1.4(.6)$ & -2.603 & .009 \\
\cline { 2 - 9 } & Afecto Negativo & 1 & 5 & $2.8(1.2)$ & $1.9(.8)$ & -2.564 & .010 \\
\cline { 2 - 9 } & Sensibilidad & 1 & 5 & $2.1(1.4)$ & $1.3(.8)$ & -2.401 & .016 \\
\cline { 2 - 9 } & Sueño & 1 & 5 & $1.7(.9)$ & $2.5(1.4)$ & -1.869 & .062 \\
\hline
\end{tabular}

Fuente: elaboración propia.

El análisis de los datos muestra igualmente una mejoría estadísticamente significativa en la competencia del alumnado $(Z=-2.959, p=.003)$, así como en todas las habilidades adaptativas evaluadas: comunicación $(Z=$ -3.576, $p=.000)$, desarrollo social $(Z=-3.622, p=.000)$, ocio $(Z=-3.542, p=.000)$, autocuidado $(Z=-3.361$, $p=.000)$, autodirección $(Z=-3.575, p=.000)$ y adaptación a la escuela $(Z=-3.662, p=.000)$. (Ved Tabla 5).

Tabla 5. Puntuaciones obtenidas en la competencia socioemocional, en sus dimensiones y en las habilidades adaptativas al inicio y al final de curso

\begin{tabular}{|c|c|c|c|c|c|c|}
\hline \multirow{2}{*}{ Variables } & \multicolumn{2}{|c|}{ Puntuaciones } & \multirow{2}{*}{$\begin{array}{c}\text { Inicio } \\
\text { M (DT) }\end{array}$} & \multirow{2}{*}{$\begin{array}{c}\text { Fin } \\
\text { M (DT) }\end{array}$} & \multicolumn{2}{|c|}{ Wilcoxon } \\
\hline & Mínima & Máxima & & & $\mathbf{Z}$ & $\mathbf{p}$ \\
\hline Comunicación & 0 & 3 & $2.05(.78)$ & $2.76(.31)$ & -3.576 & .000 \\
\hline Desarrollo social & 0 & 3 & $1.90(.45)$ & $2.52(.32)$ & -3.622 & .000 \\
\hline Ocio & 0 & 3 & $2.32(.45)$ & $2.82(.31)$ & -3.552 & .000 \\
\hline Autocuidado & 0 & 3 & $1.53(.18)$ & $2.60(.21)$ & 3.626 & .000 \\
\hline Autodirección & 0 & 3 & $1.83(.49)$ & $2.61(.36)$ & -3.575 & .000 \\
\hline Adaptación escolar & 0 & 3 & $1.66(.42)$ & $2.60(.27)$ & -3.622 & .000 \\
\hline Competencia & 1 & 5 & $3.47(.87)$ & $4.04(.70)$ & -2.959 & .003 \\
\hline Atención & 1 & 5 & $3.59(.78)$ & $4.27(.89)$ & -2.836 & .005 \\
\hline Cumplimiento & 1 & 5 & $3.59(.88)$ & $4.07(.70)$ & 2.744 & .006 \\
\hline Empatía & 1 & 5 & $2.72(1.15)$ & $3.11(.97)$ & 2.231 & .020 \\
\hline Imitación & 1 & 5 & $3.15(.89)$ & $4.48(.59)$ & 1.724 & .085 \\
\hline Motivación & 1 & 5 & $4.08(.77)$ & $4.42(.71)$ & 1.414 & .157 \\
\hline Relación Iguales & 1 & 5 & $3.06(1.14)$ & $4.06(.76)$ & -3.623 & .000 \\
\hline
\end{tabular}

Fuente: elaboración propia. 
Las puntuaciones alcanzadas en atención $(Z=2.836, p=.005)$, cumplimiento $(Z=2.744, p=.006)$, empatía $(Z=2.231, p=.020)$ y relación entre iguales $(Z=3.623, p=.000)$ al final de curso fueron significativamente superiores a las obtenidas al inicio; la imitación y el juego $(Z=1.724, p=.085)$ y la motivación $(Z=1.414, p$ $=.157)$ no experimentaron, en cambio, un cambio estadísticamente significativo.

De manera que podemos afirmar que, salvo las excepciones señaladas, los datos apoyan la posibilidad de que la primera hipótesis planteada sea cierta. No ocurre lo mismo con la segunda. El coeficiente de Spearman mostró una correlación negativa estadísticamente significativa entre las puntuaciones obtenidas al inicio de curso en el desarrollo de la comunicación, los comportamientos internalizantes $(\rho=-.669, p \leq .1)$ y la falta de regulación $(\rho=-.449, p \leq .05)$. Sin embargo, las correlaciones entre esta habilidad adaptativa, el comportamiento externalizante $(\rho=-.398, p \geq .05)$ y otras conductas $(\rho=-.466, p \geq .05)$ no fueron significativas (ved Tabla 6). Al final de curso, sólo alcanzó significación estadística la correlación entre la autorregulación y el comportamiento internalizante $(\rho=-.634, p \leq .1)$.

Por el contrario, todas las correlaciones entre las puntuaciones obtenidas al inicio (i) y al final de curso (f) en el desarrollo social y el comportamiento externalizante $\left(\rho_{\mathrm{i}}=-.511, \mathrm{p} \leq .05 ; \rho_{\mathrm{f}}=-.635, \mathrm{p} \leq .01\right)$, el comportamiento internalizante $\left(\rho_{\mathrm{i}}=-.748, \mathrm{p} \leq .01 ; \rho_{\mathrm{f}}=-.697, \mathrm{p} \leq .01\right)$, la falta de regulación $\left(\rho_{\mathrm{i}}=-.751, \mathrm{p} \leq .01, \rho_{\mathrm{f}}=\right.$ $-.540, p \leq .05)$ y otras conductas $\left(\rho_{\mathrm{i}}=-.604, \mathrm{p} \leq .01, \rho_{\mathrm{f}}=-.631, \mathrm{p} \leq .01\right)$ fueron estadísticamente significativas.

Del mismo modo, entre la autodirección y todos los dominios conductuales se obtuvieron correlaciones negativas estadísticamente significativas al inicio y al final de curso: comportamiento externalizante $\left(\rho_{\mathrm{i}}=-.655, \rho_{\mathrm{f}}\right.$ $=-.708)$, el comportamiento internalizante $\left(\rho_{\mathrm{i}}=-.574, \rho_{\mathrm{f}}=-.697\right)$, la falta de regulación $\left(\rho_{\mathrm{i}}=-.751, \rho_{\mathrm{f}}=-.540\right)$ y otras conductas $\left(\rho_{\mathrm{i}}=-.604, \rho_{\mathrm{f}}=-.631\right)$, con un $\mathrm{p} \leq .01$ para todos ellos, excepto en el caso del comportamiento internalizante al inicio de curso $(p<.05)$.

\begin{tabular}{|c|c|c|c|c|c|c|}
\hline & \multicolumn{2}{|c|}{ Comunicación } & \multicolumn{2}{|c|}{ Desarrollo social } & \multicolumn{2}{|c|}{ Autodirección } \\
\hline & Inicio & Fin & Inicio & Fin & Inicio & Fin \\
\hline C. Exteriorizante & -.398 & -.427 & $-.511^{*}$ & $-.635^{* *}$ & $-.655^{* *}$ & $-.708^{* *}$ \\
\hline C. Interiorizante & $-.669^{\star *}$ & $-.634^{* *}$ & $-.748^{* *}$ & $-697^{* *}$ & $-.574^{*}$ & $-.799^{\star \star \star}$ \\
\hline Falta Regulación & $-.449^{*}$ & -.416 & $-.751^{* *}$ & $-.540^{*}$ & $-.692^{* \star}$ & $-.768^{* *}$ \\
\hline O. Conductas & -.466 & -.488 & $-.604^{* *}$ & $-.631^{* *}$ & $-.704^{\star *}$ & $-.701^{* *}$ \\
\hline
\end{tabular}

${ }^{*} p \leq .05^{* \star} p \leq .01{ }^{\star \star *} p \leq .001$

Fuente: elaboración propia.

\section{Discusión}

El plan de apoyo positivo a la conducta es, fundamentalmente, una actuación dirigida a subsanar carencias que afectan tanto a los entornos vitales en los que se desenvuelven las personas, como a su repertorio conductual (Carr et al., 1999). Por ello, Carr et al. (1999) lo definen como el conjunto de procedimientos dirigidos 
a la creación de un entorno óptimo y a la enseñanza de habilidades comunicativas, sociales, de autocontrol y autorregulación. Ese entorno que apoya el aprendizaje y la participación incluye oportunidades para elegir, acceder a los materiales e implicarse en las actividades, una adecuada selección de las rutinas, estrategias que hacen ineficaces las conductas problemáticas, una formulación explícita de expectativas y normas de conducta y un reconocimiento frecuente de las conductas adaptativas, entre otros.

La práctica educativa de la escuela infantil en la que se ha desarrollado este estudio incluye igualmente estos componentes. Su alumnado, específicamente el que asistía al aula que atiende a los niños y niñas de dos a tres años, disminuyó de forma estadísticamente significativa la frecuencia de conductas disruptivas, así como la puntuación media obtenida en el comportamiento externalizante, la falta de regulación y otras conductas poco adaptativas; es importante destacar que este decremento en la frecuencia de comportamientos externalizantes no es usual en esta edad. Algunos estudios muestran que, hacia los tres años, la frecuencia de conductas disruptivas alcanza sus cifras más altas (Link y Angold, 2006; Röll et al., 2012), y que tiende a estabilizarse entre los 30 y los 54 meses (Campbell et al., 1991) y entre el año y medio y los seis años de edad (Crowther et al., 1981).

El nivel de desarrollo que los menores alcanzaron en competencia y en todas las habilidades adaptativas experimentó igualmente una mejoría estadísticamente significativa al final del curso. Son resultados similares a los que otros autores observan al aplicar el PAPC en la escuela infantil, generalmente con niños y niñas de tres a seis años, grupos más pequeños y ratios más reducidas (Duda et al., 2004; Fox y Hemmeter, 2009).

Se debe resaltar que a pesar de la mejoría que experimentó en la competencia socioemocional y en las habilidades adaptativas el alumnado que participó en este estudio al final de curso, el comportamiento internalizante no disminuyó de forma estadísticamente significativa. No es fácil regular el estado emocional asociado a esta conducta. La investigación muestra que la frecuencia de síntomas vinculados a comportamientos internalizantes tales como la depresión y la ansiedad parece mantenerse (Luby et al., 2009) o incrementarse en los primeros años de vida, según afirman, entre otros, Côté et al. (2009) tras realizar un seguimiento a 1759 bebés desde los cinco a los sesenta meses de edad.

En la actualidad, no hay programas dirigidos a intervenir específicamente sobre la ansiedad y los estados depresivos de menores de tres años (Côté et al., 2009) debido, entre otros, a la dificultad que hasta hace pocos años entrañaba diagnosticar estas alteraciones emocionales en los primeros años de vida (Gardner y Shaw, 2008), a que durante mucho tiempo se consideró que este tipo de estados depresivos solo podían invadir a personas que hubieran superado el nivel de desarrollo cognitivo y afectivo propio de la adolescencia (Luby et al., 2009), al estigma que nuestra sociedad suele asociar a la enfermedad mental especialmente si ocurre en la infancia, al sentimiento de culpa que genera en las familias, y a la actitud poco favorable de los profesionales sanitarios que tienden a infravalorar la importancia que tienen estos síntomas sobre los que algunos padres y madres les informan (Carter et al., 2004). Sin embargo y a pesar de esta tendencia, las puntuaciones que los menores que participaron en este estudio alcanzaron en ansiedad, al final de curso, fueron significativamente inferiores a las alcanzadas al inicio de curso.

Tampoco se observó una disminución estadísticamente significativa de la agresión. Las conductas agresivas son muy frecuentes en la primera infancia; las utilizan entre el $70 \%$ y el $80 \%$ de los niños de entre 24 y 30 meses de edad, según datos del Institut de la Statistique du Québec (Tremblay et al., 2008) para conseguir 
lo que quieren, afianzar la propia autonomía, tantear los límites del adulto y practicar habilidades sociales (Campbell et al., 2000); son conductas que tienden a mantenerse durante los primeros años de vida, según afirman Tremblay et al. (1996), (citados por Tremblay et al., 2008), tras realizar en Canadá un estudio longitudinal con 22831 niños de 2 a 11 años, y observar que la agresión empieza a disminuir a partir de los cuatro años, edad en la que consideran que se alcanza la madurez cerebral y la experiencia necesaria para controlar las rabietas.

La falta de regulación emocional (entendida como la habilidad para transformar las emociones o para desarrollar los mecanismos necesarios para gestionarlas) es un buen predictor de la agresión (Röll et al., 2012) y, sin embargo, en nuestro estudio observamos que hubo al final de curso una disminución estadísticamente significativa de la falta de regulación que no se acompañó de un decremento igualmente significativo de las conductas agresivas.

La distinción que realizan Diamond y Aspinwall (2003: 127) entre emoción y regulación emocional podría ayudar a comprender mejor estos resultados. Los autores, tras enfatizar la dificultad que entraña esta distinción, diferencian cinco componentes en la emoción: 1) el estado afectivo subjetivo, 2) la cognición y el procesamiento de la información, 3) la conducta y las manifestaciones expresivas, 4) la motivación y 5) la respuesta fisiológica; y afirman que la regulación emocional se refiere a "los procesos internos o interactivos mediante los que los individuos modulan consciente o inconscientemente uno o más componentes de la emoción, modificando bien su experiencia/conducta/expresión, o bien la situación que generó la respuesta emocional”.

El alumnado que participó en este estudio mejoró significativamente sus resultados en falta de regulación, y esta incipiente habilidad les permitió mejorar de forma estadísticamente significativa dos componentes de la emoción (de entre los que señalábamos en el párrafo anterior): la impulsividad (que podríamos quizá incluir en el segundo componente, la cognición) y la agresión entre iguales (el tercer componente), a una edad inferior a los cuatro años y, por lo tanto, inferior a la edad en la que Tremblay et al. (1996) suponían que se alcanza la maduración y la experiencia necesarias para regular la agresión. Aunque otros componentes de la emoción como el estado emocional (agresión) y la motivación no disminuyeron en este estudio de forma estadísticamente significativa.

Es importante resaltar que a pesar de que tanto la impulsividad como el afecto negativo se encuentran entre los considerados factores personales que influyen en la aparición de las conductas disruptivas (Campbell et al., 2000; Justicia et al., 2006), los menores de la escuela mejoraron sus puntuaciones en estas dimensiones de forma estadísticamente significativas al final del curso.

En la actualidad se considera que el desarrollo de la autorregulación emocional depende tanto de componentes biológicos (como, por ejemplo, del desarrollo de la corteza prefrontal) y de las características intrínsecas del individuo, como de la interacción que este establece con su medio social (Thompson, 1994) y del contexto en el que esta interacción se desarrolla.

EI PAPC plantea como principales objetivos de su intervención preventiva la optimización del entorno y la enseñanza de habilidades adaptativas. La escuela que ha participado en este estudio incluye ambos componentes en su proyecto educativo. Optimiza el entorno desarrollando las prácticas que hemos expuesto previamente y utilizando todos los recursos necesarios para hacer el entorno accesible y para facilitar que 
todo el alumnado lo comprenda y se oriente en él. Y su equipo docente actúa con la convicción de que la enseñanza facilita el desarrollo y asume la responsabilidad de enseñar a los menores a comunicarse, a relacionarse con los demás, a regularse a sí mismos y a resolver conflictos de forma adaptativa desde el inicio de la vida; recordemos que todas las habilidades adaptativas evaluadas (comunicación, desarrollo social, ocio, autocuidado, autodirección y adaptación escolar), así como la competencia socioemocional, la atención, el cumplimiento, la empatía y la relación entre iguales, experimentaron una mejoría estadísticamente significativa.

La segunda hipótesis planteaba que habría una correlación inversa entre el desarrollo de la comunicación y el lenguaje y los comportamientos problemáticos, basada en la consideración de que el lenguaje es el instrumento regulador del pensamiento y de la conducta (Luria, 1984; Vygotski, 1993). Sin embargo, los datos animan a rechazar esta hipótesis; parecen mostrar que los niños y niñas no han desarrollado todavía la capacidad para utilizar el lenguaje como instrumento regulador de su conducta. Es algo que, realmente, no debiera extrañar dado que entre los 18 meses y los tres años se desarrollan los mecanismos responsables de la gramaticalización necesarios para la construcción de la oración simple (López Ornat, 1992), por lo que parece natural que el uso de un instrumento que no se ha terminado de construir ofrezca cuanto menos algunas limitaciones incluso para regular intercambios sociales sencillos. Sin embargo, los niños y niñas del aula utilizan además del lenguaje oral signos, pictogramas y dibujos como herramientas de la mente, según recalcan Bodrova y Leong (2004) al mostrar cómo promover su uso en la etapa preescolar y primeros años de educación primaria. Se trata de mediadores que empiezan a utilizarse en esa fase social "intermental" que requiere la intervención del adulto para desligar la percepción (ej., del coche que tiene un amigo) de la conducta (ej., intentar cogerlo), es decir, para dar origen a la acción voluntaria (Luria, 1984; Vygotski, 1993).

Por el contrario, el análisis de los resultados mostró una correlación negativa estadísticamente significativa entre el desarrollo social y las conductas disruptivas, mostrando así un dato más que apoya el papel que pudiera tener esta dimensión en la mejora de estos comportamientos en este primer ciclo educativo, como lo mostró (entre otros) el programa de Justicia-Arráez et al. (2015) en el segundo ciclo e, igualmente, la posibilidad de mejorar esta dimensión (y el resto de las habilidades adaptativas) con un apoyo que se integra en la propia práctica educativa.

Este estudio constata igualmente una correlación negativa estadísticamente significativa entre el desarrollo de la autodirección y las conductas disruptivas. Muestra así la importancia que tiene esta dimensión, considerada uno de los componentes fundamentales de la calidad de vida y del propio desarrollo social (Tamarit, 2001; Wehmeyer y Schwartz, 1998). El empoderamiento o la percepción de sí mismo como agente de cambio constituye uno de los factores claves de la autoderminación (Wehmeyer y Mithaug, 2006). La escuela promueve esta percepción de uno mismo ofreciendo oportunidades para elegir (y para respetar las elecciones de los otros) creando un contexto que posibilita que puedan hacerlo desde muy pequeños. La correlación inversa entre esta dimensión y las conductas problemáticas alcanza los valores más altos; y correlaciona significativamente, como el desarrollo social, con todos los tipos de comportamientos desafiantes, incluyendo los comportamientos interiorizantes que se muestran tan resistentes al cambio.

Este estudio tiene algunas limitaciones. Entre ellas, se debe destacar la carencia de un grupo control, lo que impide afirmar taxativamente que la mejoría observada se debe a la metodología empleada en la escuela, y la posible influencia que la deseabilidad social pudiera haber ejercido sobre las evaluaciones que realiza 
el equipo docente de la escuela (que sin embargo no ha impedido detectar, como hemos visto, que algunas variables no experimentaron una mejoría significativa).

En cualquier caso, esta investigación tiene un carácter preliminar que invita a valorar positivamente los efectos que tiene la práctica preventiva y proactiva de una escuela infantil que educa ofreciendo un clima de convivencia positiva, guiada por los adultos, sobre la conducta, la atención, el cumplimiento, la empatía, las relaciones entre iguales y las habilidades adaptativas (comunicación, desarrollo social, ocio, autodeterminación, autocuidado, y adaptación a la escuela) y, por lo tanto, a considerar la conveniencia de utilizar, desde los primeros años de vida, metodologías similares especialmente en las escuelas que atienden a niños y niñas que presentan dificultades en el desarrollo socioemocional, o que tienen el riesgo de presentarlas. 


\section{Referencias bibliográficas}

Belsky, J. (1988): “The effect's of infant day care reconsidered”. Early Childhood Research Quarterly, 3: 235-272.

Benedict, E. et al. (2007): "Assessment and implementation of behavior supports in preschools". Topics in Early Childhood Special Education, 27 (3): 174-192.

Bennett, K. et al. (1998): "Do measures of externalizing behaviour in normal populations predict later outcome? Implications for targeted preventions to prevent conduct disorder". Journal of Child Psychology and Psychiatry, 39: 1059-1070.

Bodrova, E. y Leong, D. (2004): Herramientas de la mente. El aprendizaje en la infancia desde la perspectiva de Vygotsky. Naucalpan: Pearson Educación de México.

Campbell, S. et al. (2000): "Early externalizing behavior problems: Toddlers and preschoolers at risk for later maladjustment". Development and psychopathology, 12 (3): 467-488.

Campbell, S. et al. (1991): "Hard to manage preschool boys: family context and stability of externalizing behaviours". Journal of Abnormal Child Psychology, 19 (3): 301-318.

Carr, E. et al. (1999): Positive behavior support for people with developmental disabilities. A research synthesis. Washington, D.C.: American Association of Mental Retardation.

Carter, A. et al. (2004): "Assessment of young children's social-emotional development and psychopathology: recent advances and recommendations for practice". The Journal of Child Psychology and Psychiatry, 45 (1): 109-134.

Carter, A. et al. (2003): "The Infant-Toddler Social and Emotional Assessment Examiners (ITSEA): factor structure, reliability, and validity". Journal of Abnormal Child Psychology, 31 (5): 495-514.

Conroy, M. et al. (2005): "A descriptive analysis of behavioral intervention research with young children with challenging behavior". Topics in Early Childhood Special Education, 25: 157-166.

Côté, S. et al. (2009): "Depression and anxiety symptoms: onset, developmental course and risk factors during early childhood". Journal of Child Psychology and Psychiatry, 50 (10): 1201-1208.

Crowther, J. et al. (1981): "The incidence, prevalence and severity of behavior disorders among preschool-aged children in day care". Journal of Abnormal Child Psychology, 9: 23-42.

Diamond, L. y Aspinwall, L. (2003): "Emotion regulation across the life span: an integrative perspective emphasizing self-regulation, positive affect, and dyadic processes”. Motivation and Emotion, 27 (2): 125-156.

Duda, M. et al. (2004): "An experimental evaluation of positive behavior support in a community preschool program". Topics in Early Childhood Special Education, 24 (3): 143-155.

Dunlap, G. et al. (2006): "Prevention and intervention with young children's challenging behavior: Perspectives regarding current knowledge". Behavioral Disorders, 32 (1): 29-45.

Dunlap, G. y Fox, L. (2009): “Positive behavior support and early intervention”, en Sailor, W. et al. (coords.): Handbook of positive behavior support. Issues in Clinical. Boston: Springer.

Escribano, L. (2014): “Apoyo conductual positivo”. En Herramientas para la intervención. Ponencia llevada a cabo en las III Jornadas Autonómicas TEA, 28-29 de noviembre. Consejería de Educación Cultura y Deportes de Castilla-La Mancha, Albacete (España). 
Escribano, L. et al. (2014): "La aplicación de un plan de apoyo positivo en el contexto escolar”. Revista Española de Orientación y Psicopedagogía, 25 (3): 74-89.

Fox, L. et al. (2003): "The teaching pyramid. A model for supporting social competence and preventing challenging behaviour in young children". Young children, 58 (4): 48-52.

Fox, L. et al. (2002): "Young children with challenging behaviour. Issues and considerations". Journal of Positive Behavior Interventions, 4 (4): 208-217.

Fox, L. et al. (2001): "Developing school-wide behavior support in a community preschool". Journal of Positive Behavior Interventions, 3 (4): 251-254.

Fox, L. y Hemmeter, M. L. (2009): "A program-wide model for supporting social emotional development and addressing challenging behavior in early childhood settings", en Sailor, W. et al. (coords.): Handbook of Positive Behavior Support. Nueva York: Springer.

Gardner, F. y Shaw, D. (2008): "Behavioral problems of infancy and preschool children (0-5)", en Rutter, M. et al. (eds.): Rutter's child and adolescent psychiatry. Londres: Blackwell.

González del Yerro, A. et al. (2010): "La prevención de conductas desafiantes en la Escuela Infantil: Análisis de los efectos de un programa de formación docente", en Gázquez, J. y Pérez, C. (coords.): Investigación en Convivencia Escolar: Variables Relacionadas. Almería: GEU.

Hausfather, A. et al. (1997): "Effects of age of entry, day-care quality, and family characteristics on preschool behavior". Journal of Child Psychology and Psychiatry and Allied Disciplines, 38: 441-448.

Hemmeter, M. L. et al. (2007): "A program-wide model of positive behavior support in early childhood settings". Journal of Early Intervention, 29 (4): 337-355.

Justicia, F. et al. (2006): "Aproximación a un nuevo modelo explicativo del comportamiento antisocial”. Electronic Journal of Research in Educational Psychology, 4 (9): 131-150.

Justicia-Arráez, A. et al. (2015): "Estudio longitudinal de los efectos del programa Aprender a convivir en la competencia social infantil. Revista de Psicodidáctica, 20 (2): 263-283.

Linaza, J. y Maldonado, A. (1987): Los juegos y el deporte en el desarrollo psicológico del niño. Barcelona: Anthropos.

Link, H. y Angold, A. (2006): "Common emotional and behavioral disorders in preschool children: presentation, nosology, and epidemiology". Journal of Child Psychology and Psychiatry, 47 (3/4): 313-337.

López Ornat, S. (1992): "Sobre la gramaticalización. Prototipos para la adquisición de la concordancia verbosujeto: datos de la lengua española en niños de 1;6 a 3;6". Cognitiva, 4 (1): 49-74.

Luby, J.L. et al. (2009): "Preschool depression homotypic continuity and course over 24 months". Archives of General Psychiatry, 66 (8): 897-905.

Luria, R. (1984): Conciencia y lenguaje. Madrid: Visor.

Marchand, J. et al. (2002): "Mutual relations between mothers' depressive symptoms and hostile-controlling behavior and young children's externalizing and internalizing behavior problems". Parenting, Science and Practices, 2 (4): 335-353.

Moffitt, T. E. (1993): “Adolescence-limited and life-course-persistent antisocial behavior: A developmental taxonomy”. Psychological Review, 100: 674-701. 
Monjas, I. y González, B. (1998): Las habilidades sociales en el currículo. Madrid: Ministerio de Educación, Cultura y Deporte.

Montero, D. y Fernández-Pinto, I. (2013): ABAS-II. Sistema de Evaluación de la Conducta Adaptativa (adaptación española). Madrid: TEA Ediciones.

Peisner-Feinberg, E. et al. (2001): "The relation of child-care quality to children's cognitive and social developmental trayectories through second grade”. Child development, 72 (5): 1534-1553.

Piaget, J. (1984): El criterio moral en el niño. Barcelona: Martínez Roca.

Phillips, D. et al. (1987): "Child-care quality and children's social development”. Developmental Psychology, 23 (4): 537-543.

Pichardo, M. C. et al. (2016): "Desarrollo de la competencia social y prevención de problemas de conducta en el aula infantil”. Pensamiento Psicológico, 14 (1): 21-31.

Powell, D. et al. (2006): "Prevention and intervention for the challenging behaviors of toddlers and preschoolers". Infants and Young Children, 19 (1): 25-35.

Powell, D. y Dunlap, G. (2009): Evidence-based social-emotional curricula and intervention packages for children 0-5 years and their families (roadmap to effective intervention practices). Tampa, Florida: University of South Florida, Technical Assistance Center on Social Emotional Intervention for Young Children.

Richman, M. et al. (1982): Preschool to school: A behavioural study. London: Academic Press.

Röll, J. et al. (2012): "Emotion regulation and childhood aggression: Iongitudinal associations". Child Psychiatry \& Human Development, 43 (6): 909-923.

Sanders, M. (2008): "Triple P-Positive Parenting Program as a Public Health Approach to Strengthening Parenting”. Journal of Family Psychology, 22 (3): 506-517.

Schaeffer, B. et al. (2005): Habla signada para alumnos no verbales. Madrid: Alianza.

Shaw, D. et al. (2009): "Improvements in maternal depression as a mediator of intervention effects on early childhood problem behavior". Developmental Psychopatology, 21 (2): 417-439.

Shaw, D. y Taraban, L. (2017): "New directions in preventing conduct problems in early childhood". Child Developmental Perspectives, 11 (2): 85-89.

Schuhmann, E. M. et al. (1998): "Efficacy of parent-child interaction therapy: Interim report of a randomized trial with short-term maintenance". Journal of clinical child psychology, 27 (1): 34-45.

Tamarit, J. (2001): "Propuestas para el fomento de la autodeterminacion en personas con autismo y retraso mental”, en Verdugo, M. A. y Jordán de Urríes, B. (eds.) (2001): Apoyos, autodeterminación y calidad de vida. Salamanca: Amarú.

Tamarit, J. et al. (1990): P.E.A.N.A. Proyecto de Estructuración Ambiental en el aula de Niños/as con Autismo. Madrid: Consejería de Educación de la Comunidad de Madrid y Dirección General de Renovación Pedagógica del MEC.

Thompson, R. A. (1994): "Emotion regulation: A theme in search of definition". Monographs of the Society for Research in Child Development Monographs, 59: 25-52.

Tremblay, R. et al. (2008): Early learning prevents youth violence. Montreal, Quebec: Centre of Excellence for Early Childhood Development. 
Tremblay, R. et al. (2005): "Physical aggression during early childhood: Trajectories and predictors". Journal of the Canadian Academy of Child and Adolescent Psychiatry, 14 (1): 3-9.

Tremblay, R. et al. (1996): "Do children in Canada become more aggressive as they approach adolescence?" en Human Resources Development Canada, Statistics Canada (eds.): Growing up in Canada: National Longitudinal Survey of Children and Youth. Ottawa: Statistics Canada.

Vygotski, L. S. (1993): "Pensamiento y lenguaje”, en Vygotski, L. S.: Obras escogidas. Tomo II. Madrid: Aprendizaje Visor.

Webster-Stratton, C. (1982): "Teaching mothers through videotape modelling to change their children's behavior". Journal of Pediatric Psychology, 7: 279-294.

Wehmeyer, M. L. y Mithaug, D. (2006): “Self-determination, causal agency, and mental retardation”. International Review of Research in Mental Retardation, 31: 31-71.

Wehmeyer, M. L. y Schwartz, M. (1998): "The relationship between self-determination and quality of life for adults with mental retardation”. Education and Training in Mental Retardation and Developmental Disabilities, 33 (1), 3-12.

Wood, D. et al. (1976): "The role of tutoring in problem solving”. British Journal of Psychology, 66: 181-191. 